Ingeniería Investigación y Tecnología. Vol.XI. Núm.4. 2010 389-402,

ISSN 2594-0732, FI-UNAM (artículo arbitrado)

DOI: http://dx.doi.org/10.22201/fi.25940732e.2010.11n4.033

INVESTIGACION

inge ría

\title{
Aplicación de un filtro activo de corriente para mejorar la calidad de la energía en un sistema eléctrico industrial
}

\section{Improving Industrial Energy Quality by an Active Current Filter}

\author{
Reyes-Trujillo E. \\ Instituto de Investigaciones Eléctricas \\ Cuernavaca, Morelos, México \\ E-mail:ereyes@iie.org.mx \\ Rodríguez-Rivas J.J. \\ Sección de Estudios de Posgrado e Investigación \\ Escuela Superior de Ingeniería Mecánica y Eléctrica ESIME-Zacatenco \\ Instituto Politécnico Nacional, México \\ E-mail:jjrodriguezr@ipn.mx \\ Robles-García J. \\ Sección de Estudios de Posgrado e Investigación \\ Escuela Superior de Ingeniería Mecánica y Eléctrica, ESIME-Zacatenco \\ Instituto Politécnico Nacional, México \\ E-mail:jarobles@ipn.mx
}

(Recibido: junio de 2007; reevaluado: mayo de 2008 y junio de 2009; aceptado: abril de 2010)

\section{Resumen}

Con el incremento de las cargas no lineales en las aplicaciones industriales, surgen problemas de distorsión armónica en las señales de voltaje y de corriente, lo cual provoca pérdidas de energía y empeoramiento del factor de potencia, afectando la calidad de la energía en los sistemas eléctricos industriales. Para resolver estos problemas se han utilizado entre otros métodos, arreglos de capacitores y reactores conocidos como filtros pasivos, los cuales pueden presentar problemas de resonancia con la impedancia de la red, sobre compensación de la potencia reactiva a la frecuencia fundamental y una pobre flexibilidad dinámica para compensar diferentes componentes de frecuencias armónicas. Como una solución a los problemas mencionados, se han desarrollado los filtros activos, cuyas características se adaptan de forma dinámica a las necesidades del sistema a compensar. En este artículo se presentan el modelado y los resultados de la simulación de un filtro activo de corriente utilizado para reducir el contenido armónico y mejorar el factor de potencia de un sistema eléctrico industrial. Como carga no lineal se utilizó un convertidor de seis pulsos a diodos con carga RL (carga pasiva). Durante el análisis, se observó que el contenido total de corrientes armónicas THD se reduce para este caso de $16.47 \%$ a $0.46 \%$, y el factor de potencia en el bus de distribución se mejora de 0.5 a 0.95. Además, se muestran las formas de ondas cuando se utiliza una carga activa formada por un convertidor de seis pulsos a tiristores con una máquina de CD.

Descriptores: filtros activos de corriente, armónicos, calidad de la energía. 


\begin{abstract}
The growing number of non-linear loads on industrial applications has produced an important impact on the quality of electric power supply due to the increasing of the voltage and current harmonic distortion, and low power factor. In order to solve this, arrangements of capacitors and reactors, known as passive filters have been used. However these filters may produce resonance problems with network impedance, over compensation of reactive power at fundamental frequency, and poor flexibility for dynamic compensation of different frequency harmonic components. As a solution to the problems mentioned above, the active filters have been developed, whose features can be adapted in a dynamic and adjustable way on the requirements of the system to compensate. This paper presents the modelling and simulation results of an active current filter, used to reduce the harmonic distortion and to improve the power factor in an electric industrial system. A six-pulse diode converter has been used as non-linear passive load. During the analysis, it was observed that the total current harmonic distortion (THD) was reduced from $16.47 \%$ to $0.46 \%$, and the power factor in the distribution bus has improved from 0.5 to 0.95.The waveforms of a three-phase thyristor converter with a DC machine as active non-linear load are shown.
\end{abstract}

Keywords: Active current filter, harmonics, power quality.

\section{Introducción}

Actualmente, los problemas en la calidad de la energía eléctrica se han acentuado en las instalaciones eléctricas industriales, debido principalmente al incremento del uso cada vez mayor de la electrónica de potencia para el control de los procesos o cargas. Los dispositivos de electrónica de potencia utilizados en el control de los procesos, tienen un comportamiento no lineal, es decir, la señal de corriente es no sinusoidal cuando se le aplica un voltaje sinusoidal (IEEE Std., 519, 1992).

Esta clase de cargas contribuyen de manera significativa a degradar la calidad de la energía eléctrica utilizada, ya que generan armónicos de corriente que pueden provocar distorsión en la señal de voltaje y pérdidas (Wagner et al., 1993).

Considerando la relación costo-beneficio, el uso de este tipo de cargas es incuestionable, ya que proporcionan una mayor flexibilidad en el control de los procesos electros energéticos incrementándose su viabilidad económica.

Sin embargo, un problema importante que surge debido a su uso es el intercambio de energía reactiva con el sistema de transmisión y en muchos casos, se presentan corrientes con una forma de onda discontinua durante los semiperiodos de la frecuencia fundamental del sistema (Nuñez, 2002).

Algunos de los efectos adversos producidos por las cargas no lineales en la calidad de la energía son (Wagner et al., 1993):
- Distorsión de la señal de voltaje dentro de las instalaciones.

- La circulación de corrientes por el neutro.

- Los altos niveles de voltaje de neutro a tierra.

- Sobrecalentamientos en transformadores.

- Los grandes campos magnéticos generados en los transformadores.

- Posible reducción en la capacidad de distribución.

- Bajo factor de potencia debido a su reducción como consecuencia de los armónicos.

Los efectos mencionados se manifiestan de diferentes formas en los distintos equipos instalados en los sistemas eléctricos afectados.

Entre los efectos producidos en los equipos están los siguientes (Wagner et al., 1993):

- Calentamiento en los conductores.

- Mala operación de los interruptores y fusibles de protección.

- Operación errónea de los equipos electrónicos.

- Disminución de la vida útil en las lámparas incandescentes y riesgo de resonancia en las lámparas fluorescentes.

- Mala precisión en los dispositivos de medición ante la presencia de armónicos.

- Operación errónea de los relevadores de protección o bloqueo de los mismos ante una falla.

- Sobrecalentamiento en máquinas rotatorias. 
DOI: http://dx.doi.org/10.22201/fi.25940732e.2010.11n4.033

Reyes-Trujillo E., Rodríguez-Rivas J.J. y Robles-García J.

- Sobrecalentamiento en los devanados de transformadores y posible resonancia entre la inductancia del transformador y la capacitancia del sistema.

- Esfuerzo mecánico en aislamientos, debido a la temperatura producida por la presencia de corrientes armónicas.

Existen diferentes recursos que reducen en parte algunos de los problemas generados en la red eléctrica, pero la solución más común utilizada para filtrar armónicos y compensar el factor de potencia en aplicaciones industriales es el uso de filtros pasivos conectados en paralelo con la carga (Fang et al., 1999).

Un filtro pasivo está constituido por un arreglo de una bobina y un capacitor (LC) con baja resistencia serie para reducir pérdidas. También en algunas aplicaciones, se utilizan bancos de capacitores para mejorar el factor de potencia.

Sin embargo, en ambos casos pueden ocurrir resonancias serie o paralelo con la red, al circular a través de ella armónicos, por lo que estas distorsiones pueden ser amplificadas en vez de atenuarse con un peligro potencial de causar daños en la instalación, en los filtros LC, en los capacitores o en el propio equipo conectado a la red.

Como una alternativa a los filtros pasivos surgen los filtros activos, éstos consisten en inversores fuente de tensión o de corriente, los cuales generan las tensiones o corrientes armónicas en el mismo instante en que son demandados por la carga, cambiando constantemente su condición de operación mediante un adecuado circuito de control (Fang et al., 1999).

Las principales características de un filtro activo son: flexibilidad en el control, tiempo de respuesta rápido, bajo costo por mantenimiento (no tiene partes móviles), un equipo puede compensar varios armónicos a la vez, ya que en lugar de presentar una baja impedancia para un armónico en especial, inyecta los armónicos de corriente necesarios que demanda la carga para su adecuado funcionamiento (Wagner et al., 1993), proporciona un rango continuo de compensación de potencia reactiva y no presenta problemas de resonancia natural (Cárdenas, 1999).

En este trabajo se muestra la aplicación de un filtro activo de corriente utilizado para reducir la distorsión armónica y mejorar el factor de potencia de un sistema eléctrico industrial con una carga no lineal formada por un convertidor a diodos con una carga RL por el lado de CD (carga pasiva), también se muestran algunos resultados con un convertidor a tiristores accionando una máquina de CD (carga activa).
Considerando los resultados obtenidos, se observa que las señales de corriente se distorsionan durante la operación sin filtro y cuando se conecta el filtro activo, las señales de corriente mejoran su forma de onda considerablemente. Se presentan también los flujos de potencia en diferentes partes del sistema y se analiza la compensación del reactivo que realiza el filtro activo lográndose incrementos del factor de potencia.

\section{Modelo del filtro activo de corriente}

El filtro activo de corriente se modeló de acuerdo a cada una de las cuatro partes o etapas que lo integran, las cuales son:

\section{Etapa de adquisición de datos}

Esta etapa está formada por los transductores de voltaje y de corriente.

\section{Etapa de generación de señales de referencia}

En esta etapa se reciben las señales de la etapa anterior y se procesan para obtener las señales de referencia requeridas para la generación de los armónicos de la carga y la generación de la corriente reactiva necesaria para la compensación del reactivo, para esto se utiliza la teoría de la potencia reactiva instantánea (Akagi et al., 1984).

\section{Etapa de control}

En esta etapa se reciben las señales de corriente generadas en la etapa anterior y por medio de un control por histéresis, se generan los pulsos necesarios para activar el bloque de potencia.

\section{Etapa de potencia}

Integrada por un convertidor fuente de voltaje formado por 6 transistores de compuerta aislada (IGBT's) y un capacitor por el lado de CD; esta etapa se conecta al punto de acoplamiento común (PAC) como se observa en la figura 1.

En la figura 1 se muestran las cuatro etapas del filtro activo descritas anteriormente, obsérvese cómo en la etapa de adquisición de datos formada por transductores de corriente y de voltaje de efecto Hall, se generan señales proporcionales a los valores instantáneos de corriente y de voltaje en el punto del sistema donde se conecta el filtro, con esta etapa el circuito de generación de señales de referencias tiene la información necesaria 
para generar las señales que deben utilizarse en el circuito de control para el control de la conducción de los IGBT. A la salida del convertidor se obtendrán las componentes armónicas de corriente y las corrientes reactivas que consume la carga no lineal, evitándose que sean suministradas por el sistema de potencia.

El circuito de control también necesita las señales a la salida del filtro, por lo que es necesaria la adquisición de estas señales como se observa en la figura 1. En la etapa de control se realiza la comparación entre la señal a la salida del filtro y las señales de referencias, obteniéndose una señal de error que será utilizada por el controlador de histéresis para forzar a la corriente de salida a seguir el valor de la referencia, más adelante se explicará en detalle la operación de este lazo de control de corriente.

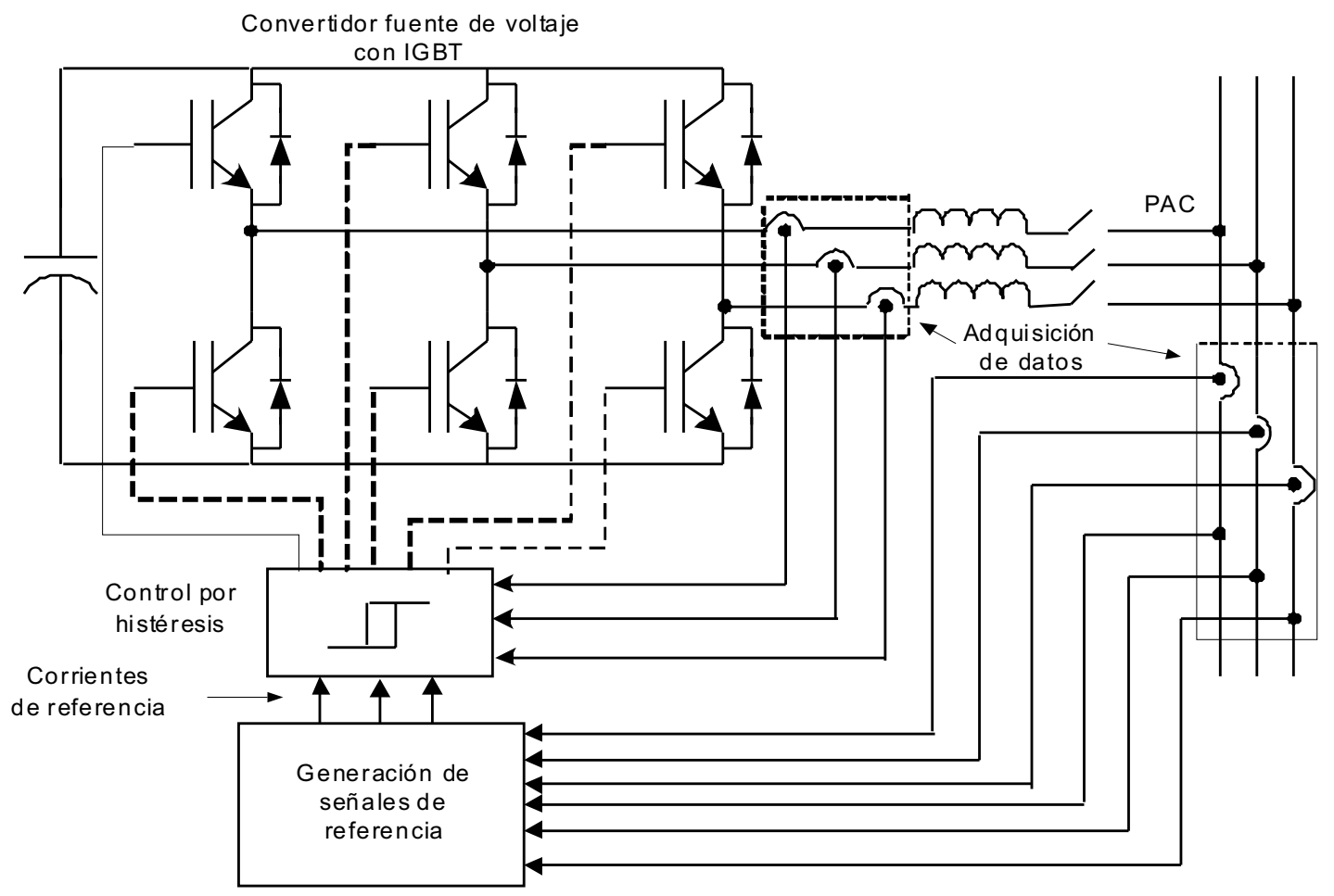

Figura 1. Diagrama general del filtro activo de corriente

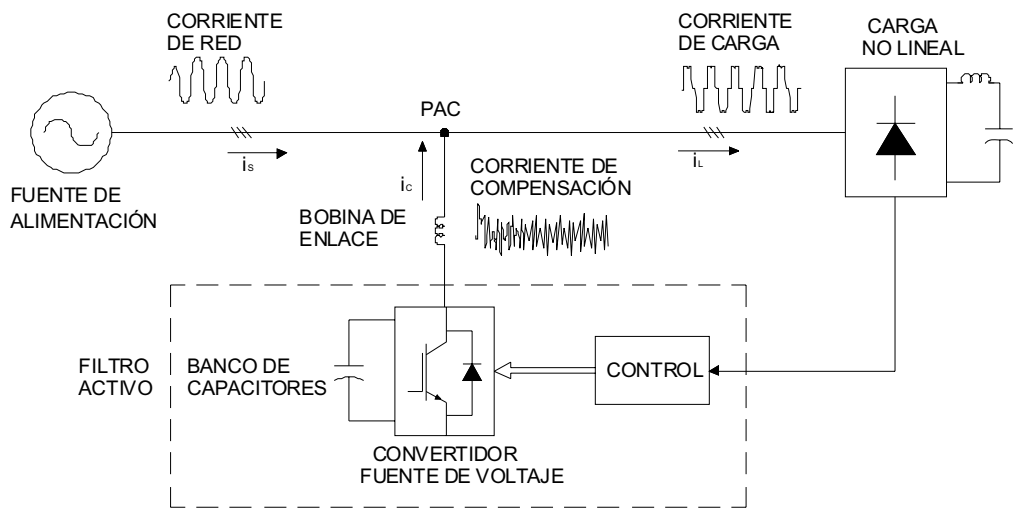

Figura 2. Principio de operación del filtro activo de corriente 
DOI: http://dx.doi.org/10.22201/fi.25940732e.2010.11n4.033

Reyes-Trujillo E., Rodríguez-Rivas J.J. y Robles-García J.

\section{Principio de operación del filtro activo de corriente}

El filtro activo de corriente se puede analizar como el elemento del sistema que genera los armónicos y la corriente reactiva presentes en la corriente de carga, evitándose de esta forma que los armónicos y el reactivo sean suministrados por el sistema. Por ejemplo, en la figura 2 se muestra la corriente de carga distorsionada, la cual tiene una componente sinusoidal que debe ser entregada por la fuente de alimentación; sin embargo, las corrientes armónicas son generadas por el filtro y suministradas a la carga por lo que no fluyen por el sistema, evitándose de esta forma, las caídas de voltajes armónicas que distorsionan la señal sinusoidal de la fuente. Como se deduce de lo anterior, el filtro debe ser conectado lo más cercano posible a la carga para evitar que los armónicos de corriente provoquen distorsión en la señal de voltaje. Para lograr lo anterior, se deben medir continuamente las corrientes de cada fase de la carga obteniéndose la componente de referencia, la cual se utilizará para generar los armónicos y la corriente reactiva que consume la carga. Esto permite que en el punto de acoplamiento común (PAC) donde es conectado el filtro activo, las corrientes armónicas que suministra la fuente de alimentación sean reducidas casi a cero y solamente sea entregada por la fuente la componente sinusoidal de la corriente de carga. En la figura 2 se observa además que el filtro activo tiene un capacitor por el lado de corriente directa, cuya función es mantener el voltaje a un determinado valor y un convertidor fuente de voltaje implementado con IGBTs. La conexión a la red eléctrica se hace a través de inductores de enlace, quedando de esta forma el filtro conectado en paralelo con la carga.

\section{Generación de las señales de referencias}

Los valores trifásicos de los voltajes y las corrientes en el punto donde se conecta el filtro (PAC), se pueden transformar a los ejes estacionarios $\alpha$ y $\beta$, utilizando la transformación a potencia constante (Krause, 1994):

$$
\begin{aligned}
& {\left[\begin{array}{l}
v_{0} \\
v_{\alpha} \\
v_{\beta}
\end{array}\right]=\sqrt{\frac{2}{3}\left[\begin{array}{ccc}
1 / \sqrt{2} & 1 / \sqrt{2} & 1 / \sqrt{2} \\
1 & -1 / 2 & -1 / 2 \\
0 & \sqrt{3} / 2 & -\sqrt{3} / 2
\end{array}\right]}\left[\begin{array}{l}
v_{a} \\
v_{b} \\
v_{c}
\end{array}\right]}
\end{aligned}
$$

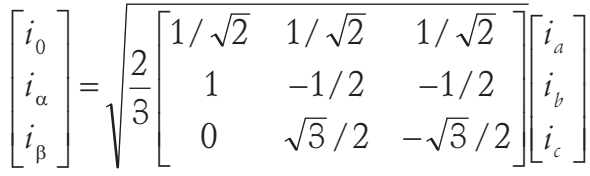
$v_{a}, v_{b}, v_{c} \quad$ Voltajes de fase.
$i_{a}, i_{b}, c_{c} \quad$ Corrientes de línea.
$i_{\alpha}, i_{\beta} \quad$ Componentes de corrientes en coordena- das estacionarias $\alpha$ y $\beta$.
$v_{\alpha}, v_{\beta} \quad$ Componentes de voltajes en coordenadas estacionarias $\alpha$ y $\beta$.
$v_{0}, i_{0} \quad$ Voltaje y corriente de secuencia cero.

En la figura 3 se observan las componentes de corrientes y de voltajes después de la transformación.

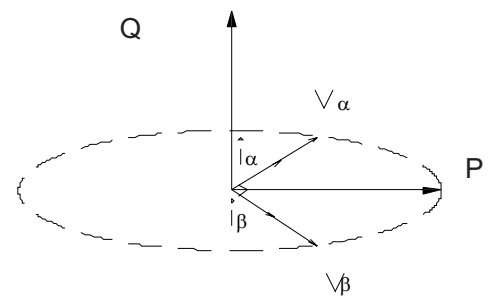

Figura 1. Potencia imaginaria instantánea

La potencia debida a los términos de secuencia cero y la potencia trifásica instantánea convencional se definen como:

$$
\begin{aligned}
& p_{0}(t)=v_{0}(t) i_{0}(t) \\
& p(t)=v_{\alpha} i_{\alpha}+v_{\beta} i_{\beta}
\end{aligned}
$$

Akagi et al., introducen el concepto de potencia instantánea imaginaria en 1984, donde definieron el vector espacial potencia imaginaria como (figura 3 ):

$$
\bar{q}=\bar{v}_{\alpha} \overline{x i}_{\beta}+\bar{v}_{\beta} \overline{x i}_{\alpha}
$$

Entonces, los valores instantáneos de la potencia de secuencia cero, de la potencia convencional y de la potencia imaginaria pueden ser expresados en forma matricial:

$$
\left[\begin{array}{l}
p_{0} \\
p \\
q
\end{array}\right]=\left[\begin{array}{ccc}
v_{0} & 0 & 0 \\
0 & v_{\alpha} & v_{\beta} \\
0 & -v_{\beta} & v_{\alpha}
\end{array}\right]\left[\begin{array}{l}
i_{0} \\
i_{\alpha} \\
i_{\beta}
\end{array}\right]
$$

De la ecuación (6) se obtiene que los valores instantáneos $p$ y $q$ tienen componentes de corriente directa $\left(p^{C D}, q^{C D}\right)$ y de corriente alterna $\left(p^{C A}, q^{C A}\right)$, entonces:

$$
p=p^{C D}+p^{C A}
$$




$$
q=q^{C D}+q^{C A}
$$

donde:

$p^{C D}$ Es la componente directa de la expresión de potencia activa y corresponde al valor de potencia activa asociada a las componentes fundamentales de voltaje y de corriente.

$p^{C A} \quad$ Es el valor de potencia activa asociada a las componentes armónicas de corriente y de voltaje.

$q^{C D} \quad$ Es la componente directa de la expresión de potencia reactiva y corresponde al valor de potencia reactiva asociada a las componentes fundamentales de tensión y de corriente.

$q^{C A} \quad$ Es el valor de potencia reactiva asociada a las componentes armónicas de voltaje y de corriente.

Si se desea:

Compensar el factor de potencia: Se debe compensar el término $q^{C D},\left(q_{C}=-q^{C D}\right)$.

Compensar las corrientes armónicas: Se deben compensar los términos $p^{C A}$ y $q^{C A},\left(p_{C}=-p^{C A}, q_{C}=-q^{C A}\right)$.

Compensar ambas cosas: Se deben compensar los términos $p^{C A}, q^{C A}$ y $q^{C D},\left(p_{C}=-p^{C A}, q_{C}=-q^{C A}-q^{C D}\right)$.

Compensar el desbalance de las fases: Se debe compensar $p_{0},\left(p_{C}=-p_{0}\right)$.

Donde $p_{C}$ y $q_{C}$ son las potencias que el filtro activo debe inyectar para compensar el factor de potencia, las corrientes armónicas y/o el desbalance de fase.

El proceso de compensación debe realizarse con mucho cuidado, ya que las componentes de CA no deben ser atenuadas ni desfasadas para que a partir de ellas, se puedan calcular las corrientes de referencia de forma correcta. Por otra parte, la componente de secuencia cero debe ser compensada en su totalidad para cancelar posibles desbalances de corriente.

Sustituyendo las potencias de compensación en la ecuación (6), se pueden obtener las corrientes de compensación en los ejes ortogonales $\alpha$ y $\beta$ como sigue:

$$
\left[\begin{array}{l}
p_{C 0} \\
p_{C} \\
q_{C}
\end{array}\right]=\left[\begin{array}{ccc}
v_{0} & 0 & 0 \\
0 & v_{\alpha} & v_{\beta} \\
0 & -v_{\beta} & v_{\alpha}
\end{array}\right]\left[\begin{array}{l}
i_{0} \\
i_{\alpha} \\
i_{\beta}
\end{array}\right]
$$

Despejando se obtienen las corrientes de compensación:

$$
\left[\begin{array}{l}
i_{0 C} \\
i_{\alpha C} \\
i_{\beta C}
\end{array}\right]=\left[\begin{array}{ccc}
v_{0} & 0 & 0 \\
0 & v_{\alpha} & v_{\beta} \\
0 & -v_{\beta} & v_{\alpha}
\end{array}\right]^{-1}\left[\begin{array}{l}
p_{C 0} \\
p_{C} \\
q_{C}
\end{array}\right]
$$

Resolviendo la matriz inversa:

$$
\left[\begin{array}{l}
i_{0 C} \\
i_{\alpha C} \\
i_{\beta C}
\end{array}\right]=\frac{1}{v_{\alpha}^{2}+v_{\beta}^{2}}\left[\begin{array}{ccc}
\frac{v_{\alpha}^{2}+v_{\beta}^{2}}{v_{0}} & 0 & 0 \\
0 & v_{\alpha} & -v_{\beta} \\
0 & v_{\beta} & v_{\alpha}
\end{array}\right]\left[\begin{array}{l}
p_{0 C} \\
p_{C} \\
q_{C}
\end{array}\right]
$$

Empleando $p_{C}=-p_{0}$ y la ecuación (11) se obtiene:

$$
i_{0 C}=-i_{0}
$$

Una vez obtenidas las corrientes de referencias en los ejes ortogonales $\alpha$ y $\beta$, se aplica la transformación inversa para regresar al sistema de referencia original a, b y c de la forma siguiente:

$$
\left[\begin{array}{l}
i_{a C} \\
i_{b C} \\
i_{c C}
\end{array}\right]=\sqrt{\frac{2}{3}}\left[\begin{array}{ccc}
1 / \sqrt{2} & 1 & 0 \\
1 / \sqrt{2} & -1 / 2 & \sqrt{3} / 2 \\
1 / \sqrt{2} & -1 / 2 & -\sqrt{3} / 2
\end{array}\right]\left[\begin{array}{l}
i_{0 C} \\
i_{\alpha C} \\
i_{\beta C}
\end{array}\right]
$$

Siendo:

$i_{a C}$ Corriente de compensación de la fase a usada como referencia.

$i_{b C}$ Corriente de compensación de la fase $b$ usada como referencia.

$i_{c C}$ Corriente de compensación de la fase c usada como referencia.

Considerando el procesamiento matemático anterior, en la figura 4 se muestra el programa en Simulink para calcular las corrientes de referencias que se generan para eliminar los armónicos, compensar el reactivo y en caso necesario, balancear el sistema. Estas corrientes de referencia son suministradas al bloque de control

\section{Control de las corrientes de compensación}

En la figura 5a se muestra el principio de operación del control de las corrientes de compensación, utilizando un controlador de histéresis. Primero se comparan las señales de referencias con las señales reales a la salida 
del convertidor fuente de voltaje, el valor del error debido a la comparación, entra al controlador de histéresis como se puede observar en la figura $5 \mathrm{~b}$. La salida del controlador genera los pulsos de control de los transistores de compuerta aislada (IGBT) del convertidor, los cuales son modulados en el ancho de los pulsos (PWM) para forzar que la corriente de salida del convertidor siga el valor de la corriente de referencia, de esta forma se obtiene la corriente de compensación a la salida del convertidor, el cual actúa como filtro activo de armónicos y como compensador de la potencia reactiva.
En la figura 6 se muestra el programa en Simulink del sistema de control, donde se observa que después de la comparación entre las señales de referencias y las señales de compensación a la salida del filtro, se obtienen las señales de error que entran a los controladores de histéresis. La salida de los controladores son las señales de control que entran a la etapa de potencia (IGBT). En la figura se observan algunos arreglos de compuertas lógicas, las cuales se utilizan para adecuar las señales entre los bloques del Simulink y generar las señales lógicas de control de los IGBT del convertidor.

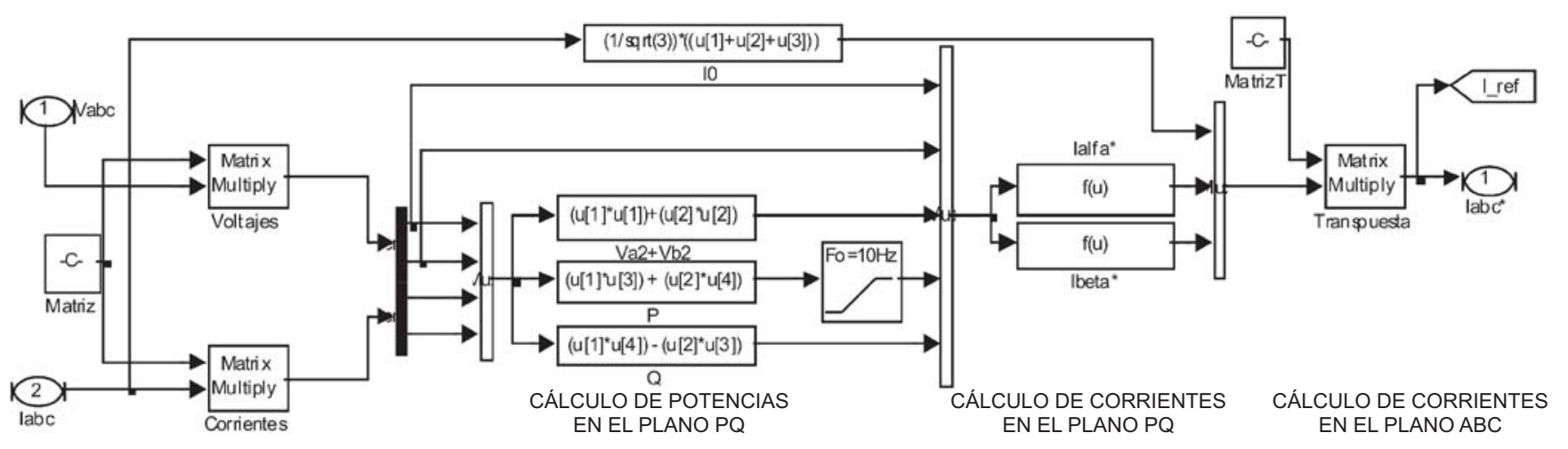

Figura 4. Programa en Simulink para el cálculo de las corrientes de referencia
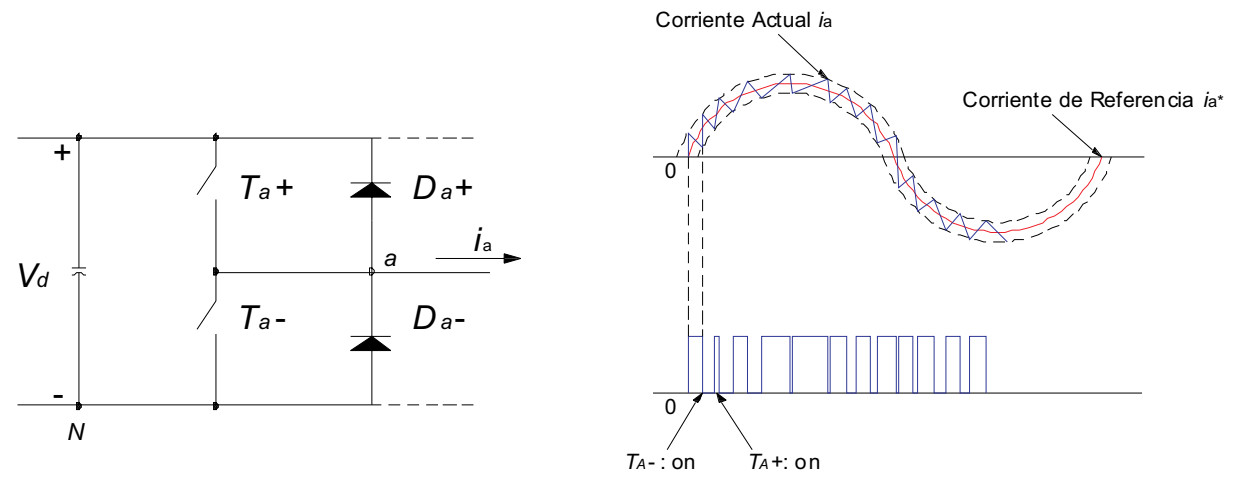

(A)

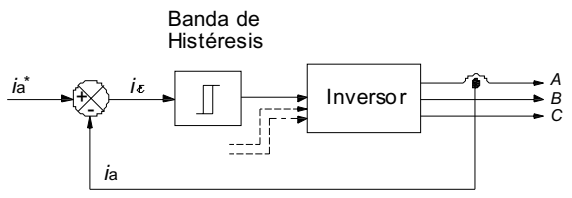

(B)

Figura 5. Controlador de corrientes por histéresis: a) Principio de operación; b) Diagrama de bloques 


\section{Simulación del sistema eléctrico}

El sistema eléctrico utilizado durante las simulaciones (sistema de prueba) se muestra en la figura 7 , el cual está integrado por un turbogenerador de 3,500 $\mathrm{kVA}$, a $4.16 \mathrm{kV}, 60 \mathrm{~Hz}$, conectado a una barra de un panel de distribución en $4.16 \mathrm{kV}$, donde se alimenta un transformador de 4 MVA, 4160/480 V, conexión delta-estrella aterrizada.

El secundario del transformador se conecta a la barra de un panel de distribución en $480 \mathrm{~V}$, donde se conectan el convertidor a diodos o tiristores según sea el caso que se analice y una carga lineal RL de $100 \mathrm{~kW}$ como se muestra en la figura 7.

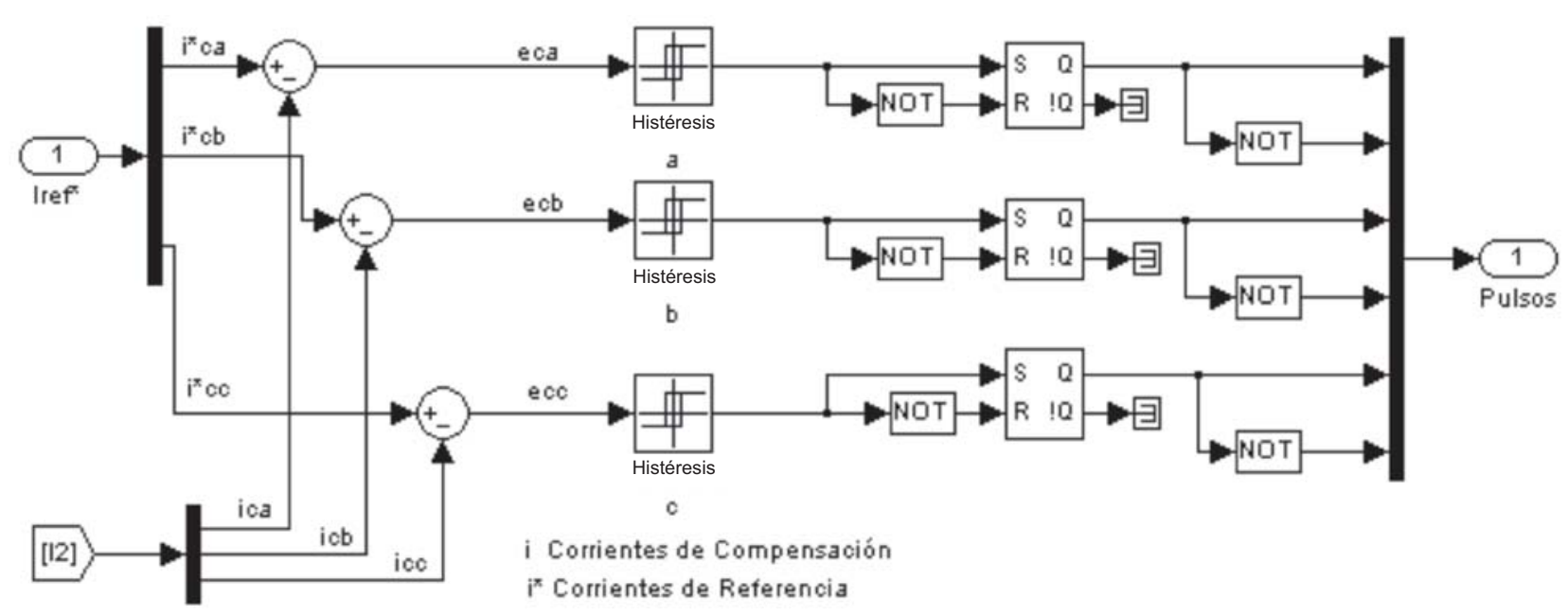

Figura 6. Control por histéresis de las corrientes de compensación

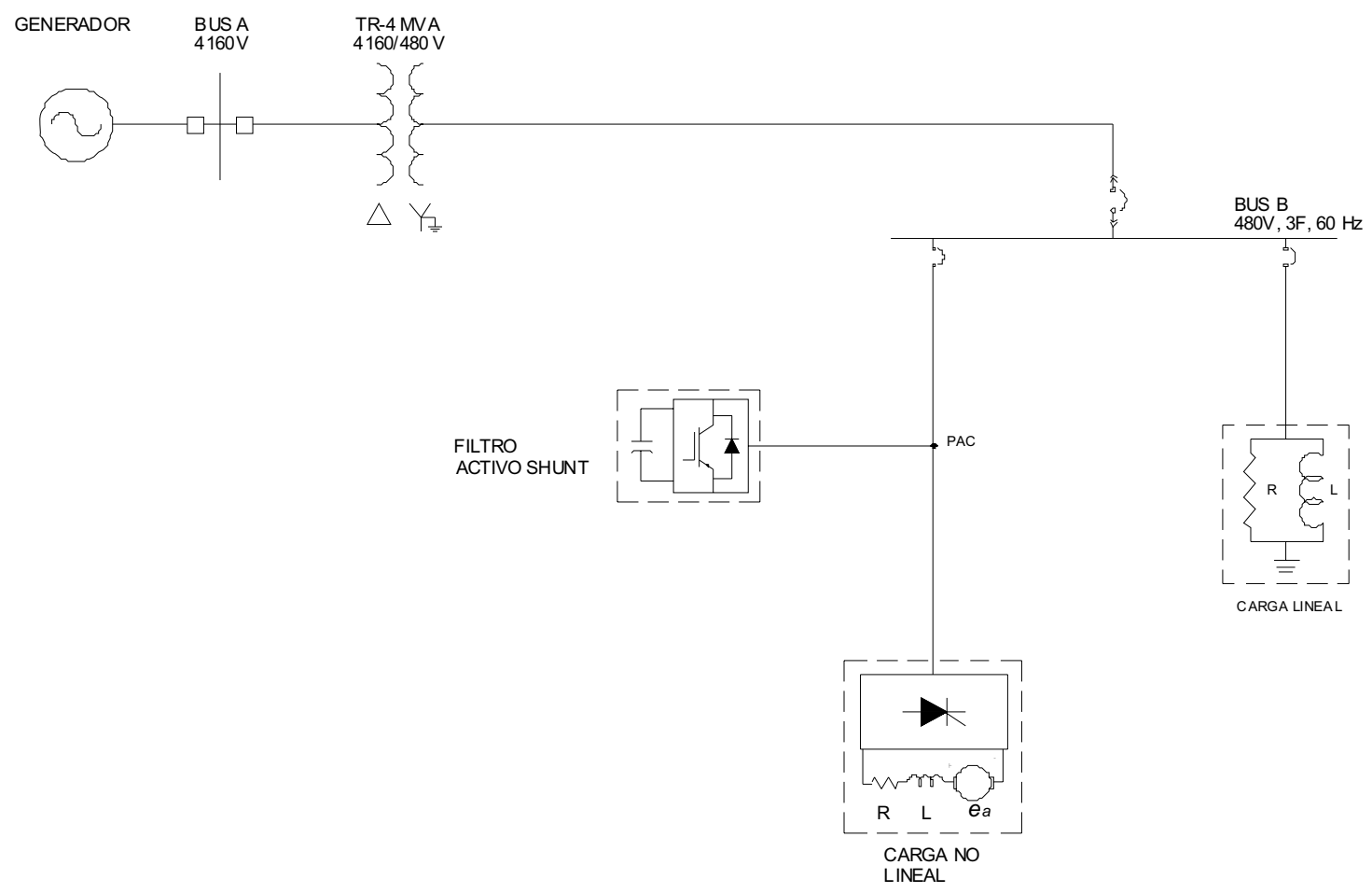

Figura 7. Sistema eléctrico industrial de prueba 
DOI: http://dx.doi.org/10.22201/fi.25940732e.2010.11n4.033

Reyes-Trujillo E., Rodríguez-Rivas J.J. y Robles-García J.

\section{Resultados}

En el sistema eléctrico mostrado en la figura 7, el filtro activo de corriente se utiliza para compensar los armónicos generados por la carga no lineal. El sistema se modeló en Simulink de Matlab como se muestra en la figura 8.

En la figura 9 , se muestran las señales de voltaje en el bus $\mathrm{B}$, donde se observa que no son afectadas por la carga no lineal, debido a la acción del filtro.

En la parte superior de la figura 10 se muestran las señales de la corriente en la carga no lineal (convertidor a diodos con carga $\mathrm{RL}$ ); en la figura del centro se muestra la corriente inyectada por el filtro activo y en la parte inferior la corriente en el bus B, es decir, en el alimentador del sistema eléctrico industrial. Después de $0.05 \mathrm{~s}$, el filtro activo comienza a compensar y se observa como las corrientes prácticamente son sinusoidales; esto significa que el filtro activo está inyectando los armónicos demandados por la carga no lineal.

En la figura 10 se observa que el filtro activo se conecta al sistema después de transcurrido un tiempo de 0.05 s. Durante este tiempo, la señal de corriente en el sistema está distorsionada como se puede apreciar en la parte inferior de la figura 10. En la figura 11, se presenta el espectro de la corriente antes de la compensación y en la figura 12 después de la compensación. La mejora en el contenido total armónico (THD) fue de un $16.47 \%$ antes de la compensación, hasta un $0.46 \%$ después de la compensación, valor inferior al exigido por la norma IEEE Std., 519, 1992.

La figura 13 muestra el comportamiento del filtro cuando se utiliza una carga no lineal formada por un convertidor a tiristores accionando una máquina de $\mathrm{CD}$. En esta figura en $0.3 \mathrm{~s}$ se conecta el filtro para compensar la carga no lineal (parte superior de la figura), la cual tiene una amplitud de unos $200 \mathrm{~A}$; treinta milisegundos después cuando aún no se alcanza el estado estable, ocurre una variación brusca de la carga no lineal, la cual se incrementa hasta una amplitud de $340 \mathrm{~A}$. En el centro de la figura se observa la variación de la corriente generada por el filtro para compensar el incremento de los armónicos y la componente reactiva de la corriente.

En la parte inferior se observa como la corriente en el alimentador del bus B es compensada lográndose alcanzar el estado estable en varios ciclos; se pueden observar pequeños picos de corriente, debido a la conmutación de los tiristores del convertidor, los cuales no aparecen en la figura 10.

Un análisis más detallado de la operación de la carga no lineal con un convertidor a tiristores se puede hallar en Reyes, 2005. También en la misma referencia se describe el diseño del filtro donde se calculan los IGBT, los reactores de enlace y el capacitor por el lado de $\mathrm{CD}$ del convertidor.

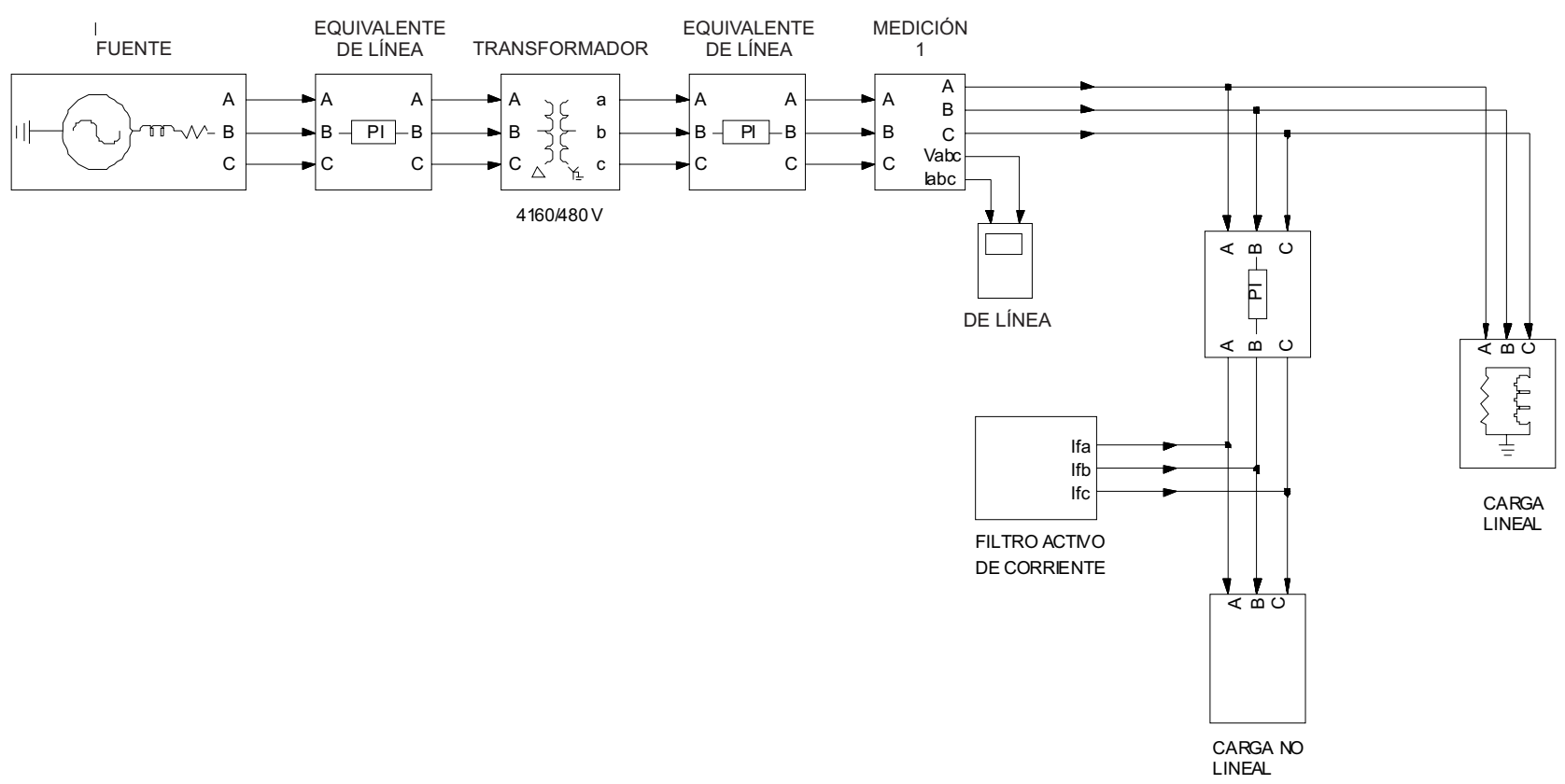

Figura 8. Sistema eléctrico industrial simulado en Simulink 

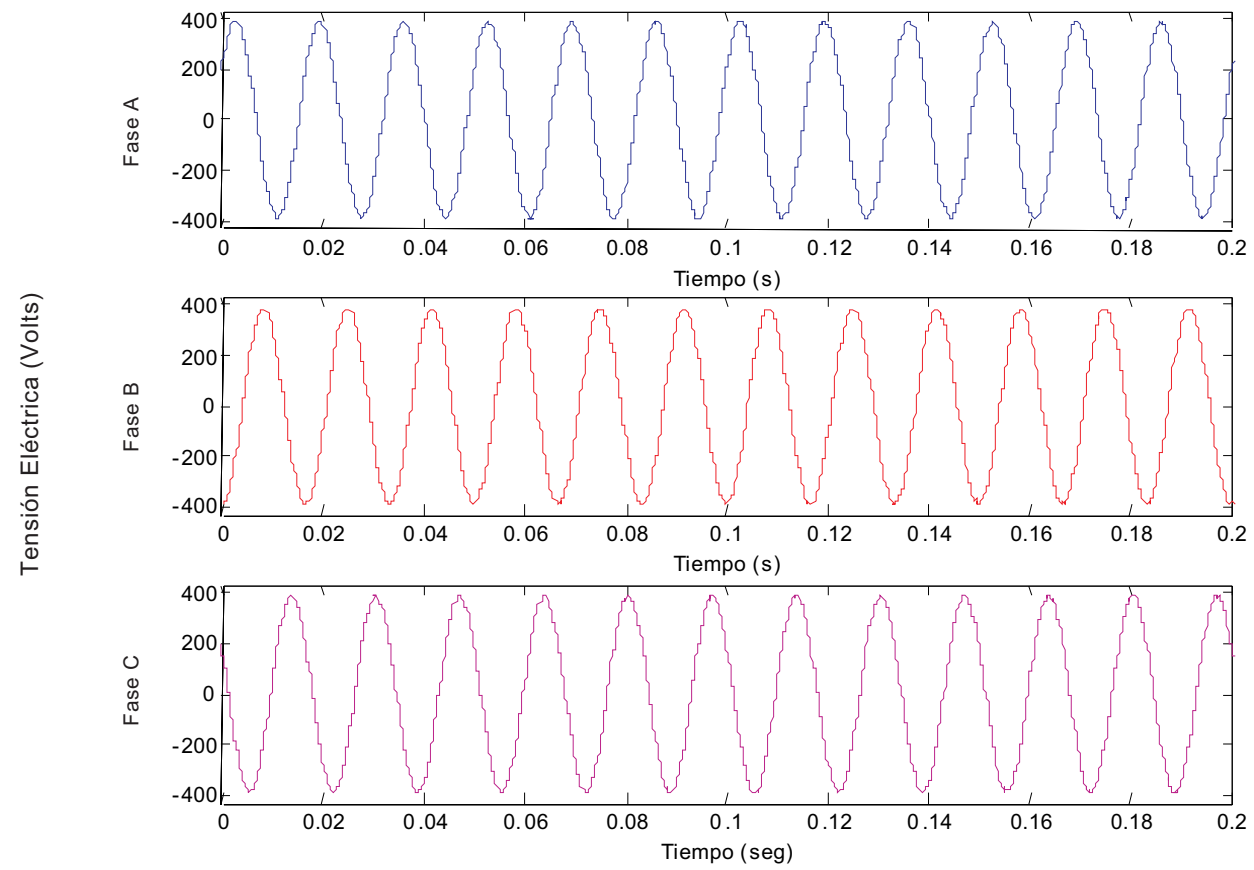

Figura 9. Señales de voltaje en el bus B obtenido de simulación
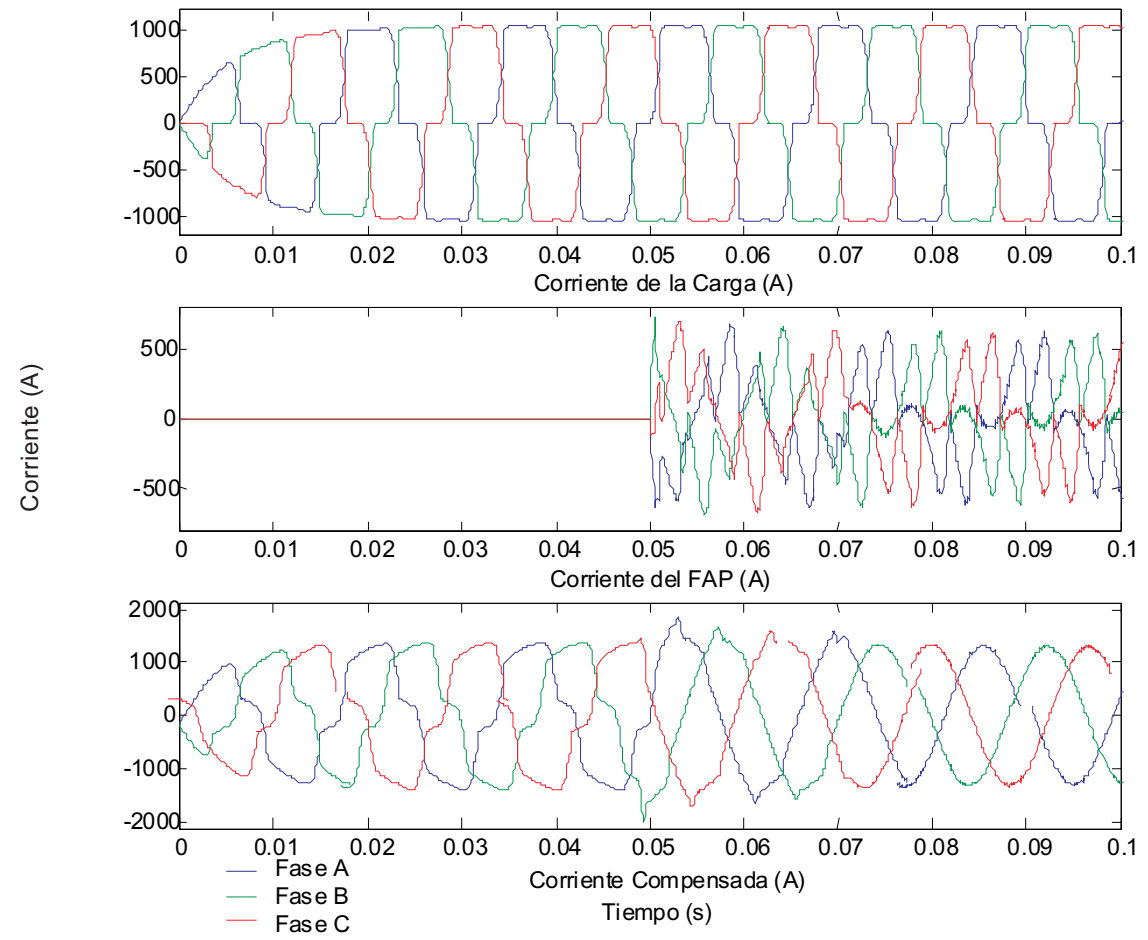

Figura 10. Señales de corriente en la carga no lineal (superior), a la salida del filtro (central) y en el bus B (inferior) 


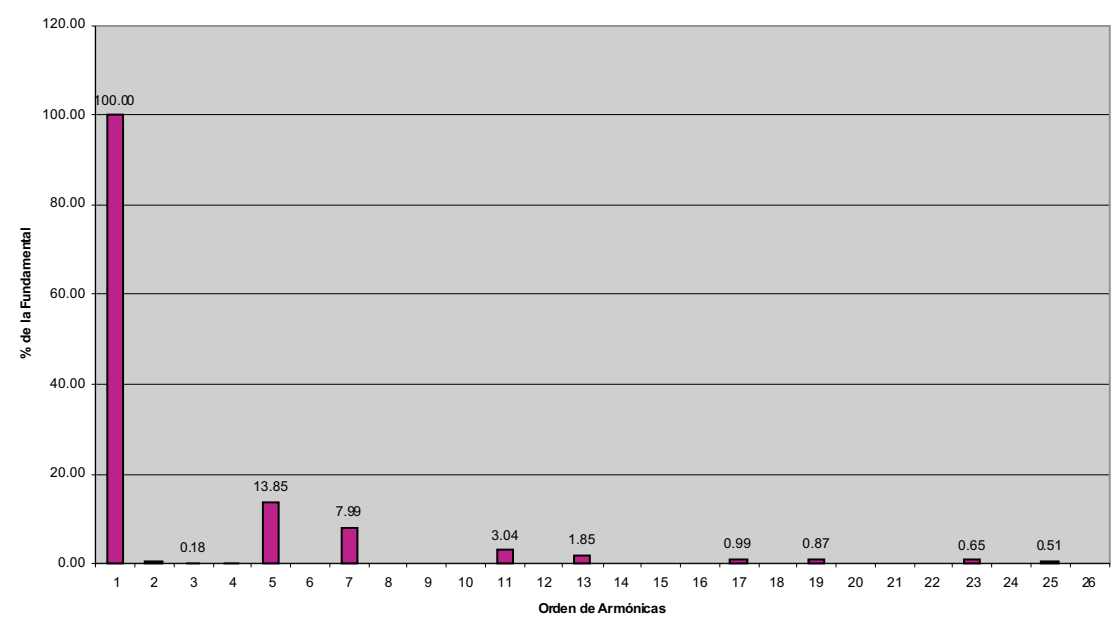

Figura 11. Espectro armónico de la corriente sin compensar

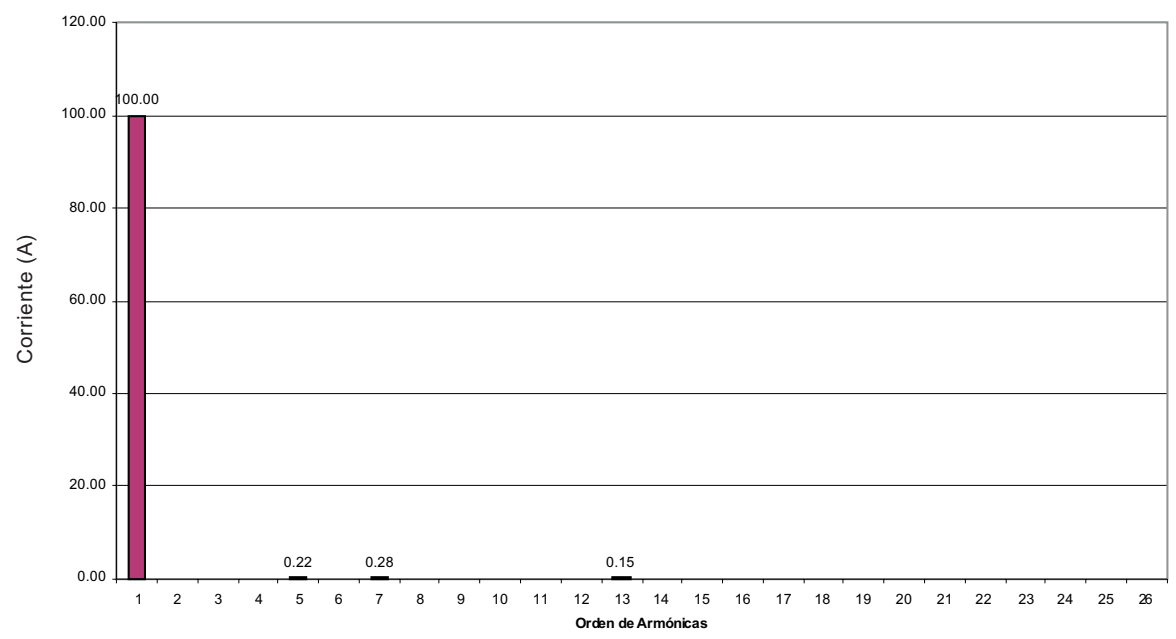

Figura 12. Espectro armónico de la corriente con compensación
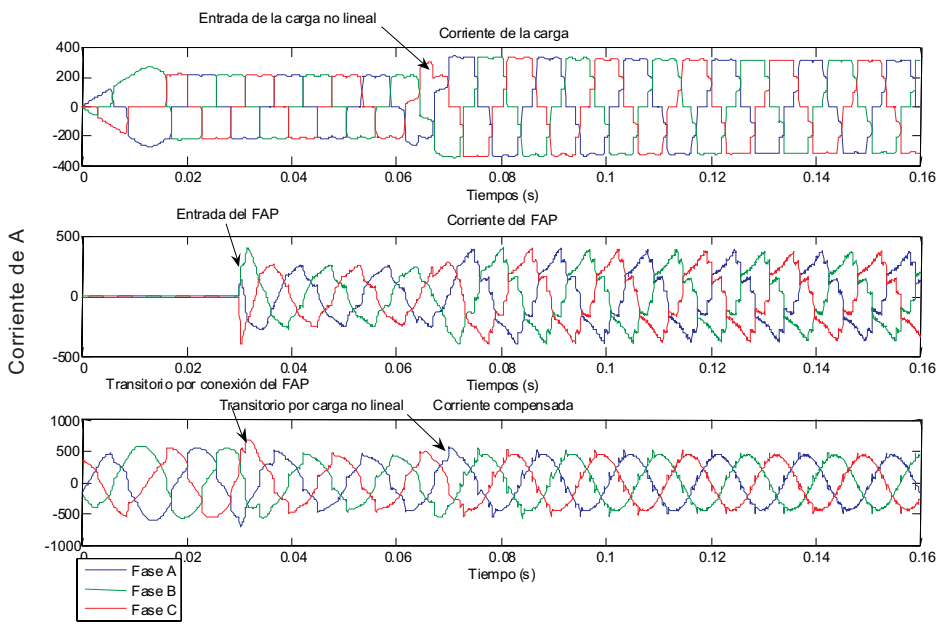

Figura 13. Comportamiento del FAP durante una variación de la carga no lineal 


\section{Factor de potencia}

En las figuras 14 y 15 se muestran los valores del factor de potencia antes y después de conectado el filtro, estos valores fueron calculados en el bus B para el caso de la carga no lineal con el convertidor a diodos. En estas figuras se observa que el filtro activo mejoró el factor de potencia de 0.42 a 0.97 . Es importante mencionar que este factor de potencia se calcula considerando las corrientes armónicas presentes en el sistema (Reyes, 2005).

\section{Flujos de potencia}

Para observar el comportamiento de los flujos de potencia, en la figura 16 se muestra el diagrama unifilar del sistema con los flujos de potencia en cada una de sus ramas. Analizando estos flujos, se observa que la demanda de la carga lineal es de $100 \mathrm{~kW}$ y $173 \mathrm{kVAR}$, lo que corresponde a una potencia total de $199.8 \mathrm{kVA}$. La demanda de potencia activa es suministrada por la fuente de generación principal, la cual entrega $734 \mathrm{~kW}$, de los cuales $624 \mathrm{~kW}$ son suministrados a la carga no lineal, $100 \mathrm{~kW}$ son suministrados a la carga lineal y 10 $\mathrm{kW}$ al filtro activo (debido a las pérdidas en los IGBT del convertidor). La fuente de alimentación principal, suministra $173 \mathrm{kVAR}$, los cuales son consumidos por la carga lineal. Los $212 \mathrm{kVAR}$ requeridos para cubrir la demanda de la carga no lineal es suministrada por el filtro activo.

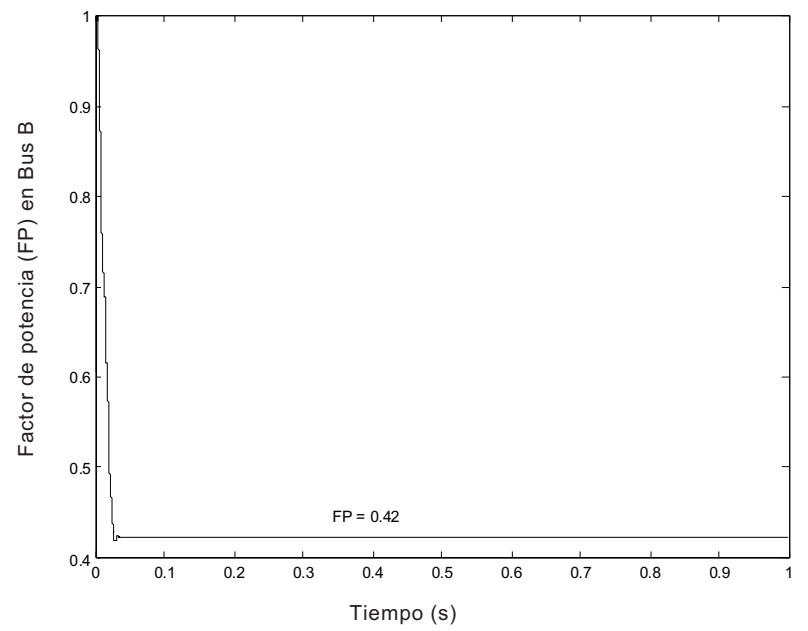

Figura 14. Factor de potencia antes de la conexión del filtro
La generación de la potencia reactiva exigida por una carga se debe realizar lo más cercano posible a dicha carga, de esta forma se reducen las pérdidas, debido a que se disminuye el flujo de corriente reactiva por el sistema de alimentación.

\section{Conclusiones}

De acuerdo a los resultados obtenidos, se puede concluir que utilizar un filtro activo de corriente en un sistema eléctrico industrial donde se tengan problemas de calidad de la energía por cargas no lineales es una buena opción, ya que permite mejorar la calidad en la señales de corriente y voltaje. Para el caso analizado, se obtuvo una reducción en la distorsión armónica total en la corriente del $16.47 \%$ al $0.46 \%$.

El factor de potencia en el bus B se elevó desde un valor de 0.42 hasta un valor de 0.97 .

Debido a que la carga no lineal está aislada de tierra, no fue necesario compensar las corrientes de secuencia cero.

Los resultados obtenidos con la instalación del filtro activo en el sistema industrial analizado permite la reducción de las pérdidas, la reducción de armónicos y la mejora en el factor de potencia del sistema industrial. Es importante mencionar que el comportamiento del filtro activo puede variar de acuerdo a la configuración del sistema eléctrico y sus cargas, por lo cual, durante el dimensionamiento del filtro se deben de tomar en cuenta las características propias de la instalación.

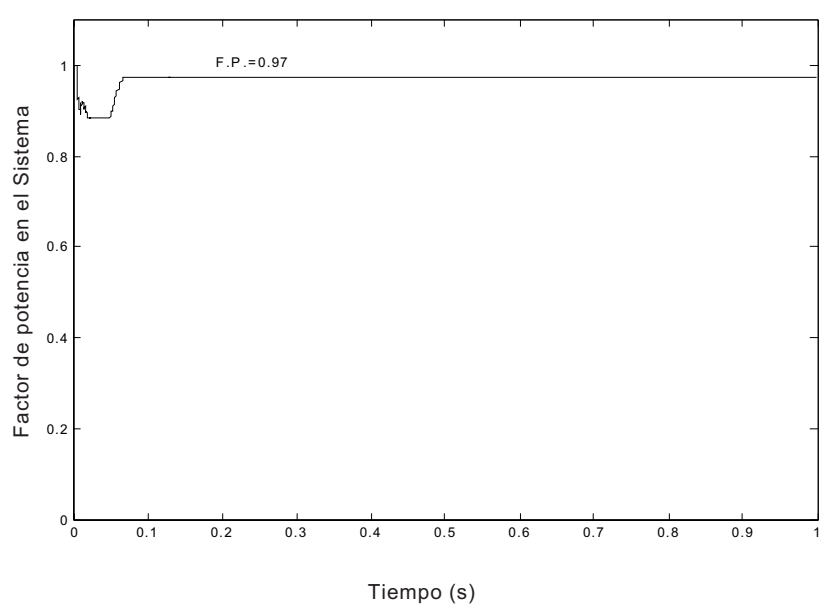

Figura 15. Factor de potencia con el filtro conectado 


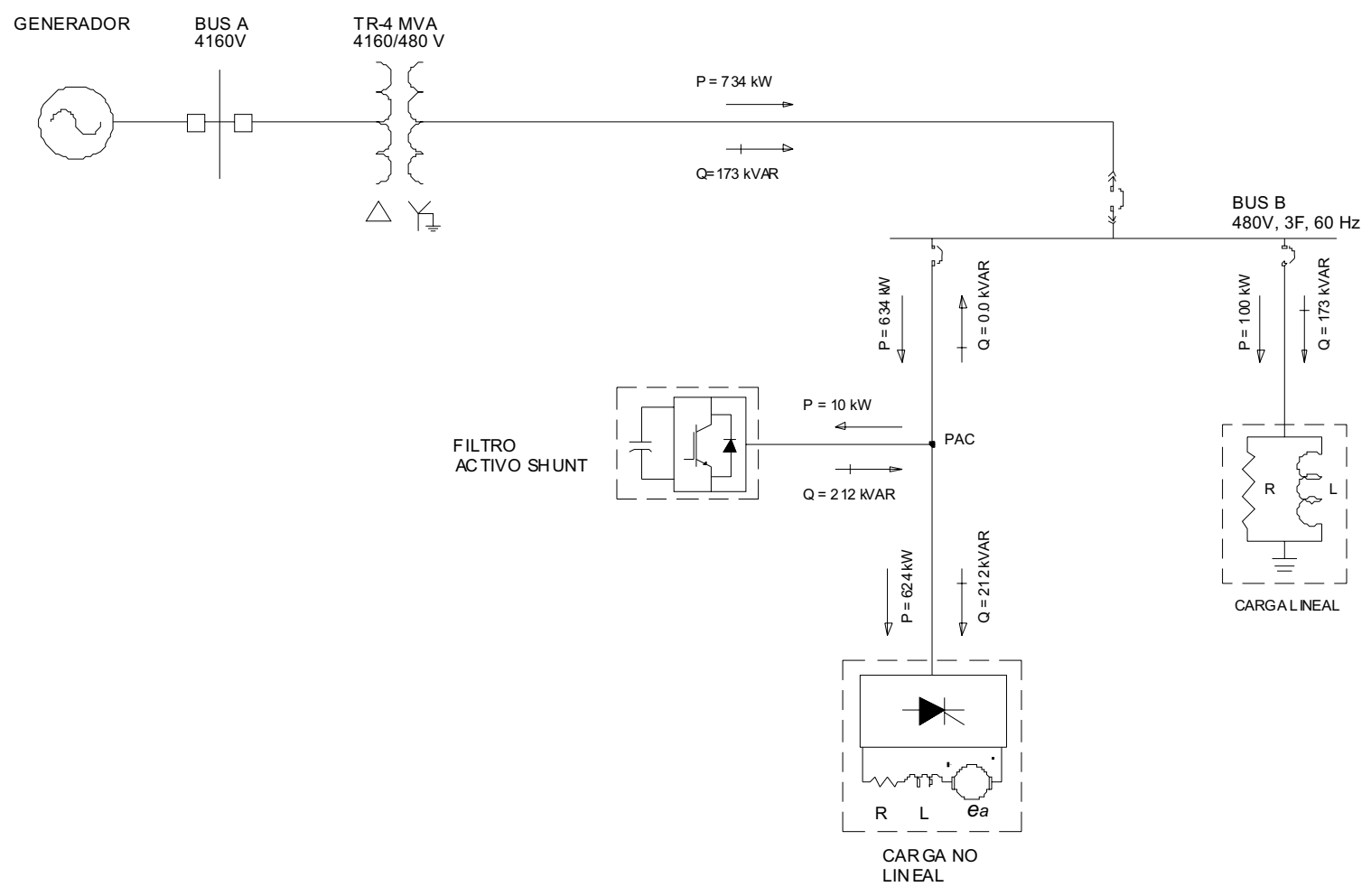

Figura 16. Flujos de potencia en las ramas del sistema eléctrico

\section{Referencias}

Akagi H., Kanazawa Y. y Nabae A. Instantaneous Reactive Power Compensators Comprising Switching Devices Without Energy Storage Components. IEEE Trans. on Industry Application, . IA-20(3):625-630, May/June, 1984.

Barrero F. Análisis topológico y funcional de acondicionadores para la reducción de perturbaciones en la red eléctrica. Tesis (Doctorado). Madrid, España. Universidad Nacional de Educación a Distancia, Escuela Técnica Superior de Ingenieros Industriales, Dpto. de Ingeniería Eléctrica, Electrónica y de Control. 1995. Pp. 4-30.

Cárdenas V. Filtros activos híbridos para compensación armónica de corriente y de compensación de factor de potencia en sistemas trifásicos. Tesis (Doctorado). Cuernavaca, México. Centro Nacional de Investigación y Desarrollo Tecnológico. 1999.
Fang Z.P., Donald J.A. Harmonic Sources and Filtering Approaches. IEEE on Industrial Application, 34th Annual Meeting. Phoenix Arizona, 1999, pp. 448-455.

Krause P.C. Analysis of Electric Machinary. IEEE Press. 1994.

IEEE Std. 519-1992. Recommended Practices and Requirements for Harmonic Control in Electrical Power Systems. IEEE Industry Applications Society.

Nuñez-Gutiérrez C.A. Estrategias de control no lineal aplicadas a filtros activos de potencia. Tesis (Doctorado). Cuernavaca, México. CENIDET. 2002.

Reyes-Trujillo E. Aplicación de un filtro activo de corriente para la reducción de armónicas y corrección del factor de potencia en sistemas eléctricos industriales. Tesis (Maestría). DF, México. ESIMEZ, IPN. 2005.

Wagner V.E., Balda et al. Effects of Harmonics on Equipment. IEEE Transactions on Power Delivery, 8(2):672-679. 1993. 
DOI: http://dx.doi.org/10.22201/fi.25940732e.2010.11n4.033

Aplicación de un filtro activo de corriente para mejorar la calidad de la energía en un sistema eléctrico industrial

\section{Semblanza de los autores}

Reyes-Trujillo Eleazar. Ingeniero electricista egresado del Instituto Tecnológico del Istmo en 1997. Obtuvo el grado de maestro en ciencias en 2005 por la Escuela Superior de Ingeniería Mecánica y Eléctrica del Instituto Politécnico Nacional. De 1998 al 2000, realizó supervisión de obras y diseño de instalaciones eléctricas. En 2001, ingresó al Instituto de Investigaciones Eléctricas en el área de uso de la energía eléctrica, donde labora actualmente. Es miembro del IEEE.

Rodríguez-Rivas Jaime José. Ingeniero electricista egresado de la Universidad Central de Las Villas (UCLV), Cuba en 1980. Obtuvo el grado de maestro en ciencias en 1987 y el de doctor en ciencias en 1991, ambos en el Instituto Energético de Moscú. Trabajó como profesor en la UCLV desde 1980 hasta 1994. Desde 1994, hasta la fecha, trabaja como profesor investigador en la SEPI, ESIMEZ, del Instituto Politécnico Nacional. Es miembro de la IEEE.

Robles-García Jaime. Ingeniero electricista egresado de la Escuela Superior de Ingeniería Mecánica y Eléctrica del Instituto Politécnico Nacional (ESIMEZ-IPN) en 1988. Obtuvo el grado de maestro en ciencias en la ESIMEZ-IPN en 1992. Obtuvo el grado de doctor en ciencias en la ESIME-IPN en 1996. Ingresó a la Comisión Federal de Electricidad en 1992, en la Gerencia de Programación de Sistemas Eléctricos, donde desempeñó el cargo de jefe de estudios dinámicos del sistema eléctrico nacional. Actualmente es profesor-investigador en la SEPI, ESIMEZ, del Instituto Politécnico Nacional. Es miembro del IEEE. 